\title{
A Global Topological Map formed by Local Metric Maps*
}

\author{
Saul Simhon
Centre for Intelligent Machines
McGill University
3480 University St.
Montreal, QC H3A 2A7

Gregory Dudek

\begin{abstract}
In this paper we describe a method of mapping large scale static environments using a hybrid topologicalmetric model. A global map is formed from a set of local maps organized in a topological structure. Each local map contains quantitative environment information using a local reference frame. They are denoted as islands of reliability because they provide accurate metric information of the environment. The mapping problem then becomes where to place the islands of reliability and to what extent should they cover the environment. This is accomplished by defining the placement criteria in terms of the task the islands of reliability portray.
\end{abstract}

\section{Introduction}

This paper addresses the issue of how and where to create nodes of a topological model of a real environment. These nodes contain geometric local maps for a dual topological-metric model. While a single metric coordinate system is a natural way to map space and is effective over small areas, over large extents of space it becomes problematic. In particular, over large regions of space incremental position errors can accrue to cause large errors in the global coordinate system. This can occur even when beacons or landmarks are used to reduce odometry error.

In many cases, it is sufficient to create local coordinate frames only in selected regions, where odometry error must be minimized. Kuipers, for example, considers this problem in the context of building topological maps and proposes using rehearsal procedures to eliminate the effect of odometric errors [1]. In our

\footnotetext{
*This paper appears in the Proceedings for the IEEE/RSJ International Conf. on Intelligent Robotic Systems, October 1998, Victoria, B.C.
}

work, we are interested in mapping using a collection of local coordinate frames organized topologically [2]. Where should we attempt to create a local metric map so that it will be accurate and effective? In this paper, we consider how to evaluate the local environment with respect to an arbitrary localization procedure. We show that we can develop a technique that predicts how appropriate a given region will be for localization (and hence for metric mapping). The specific method used to generate the local map is assumed to exist in advance. We incorporate this within mapping criteria to create a global map from a set of local maps.

\section{Background}

In order to perform accurate positioning, Dudek and Mackenzie [3] composed sonar based maps where explicit object models were constructed out of sonar reading distribution in space. The maps were used to determine robot pose by fitting new sensor data to the model. Dudek and Zhang [4] used a vision system to provide a feature based model of the environment. Position calibration was attained by interpolating through the extracted feature-pose space.

These traditional metric methods use a single reference frame. Although they provide accurate local correspondence, accumulated errors tend to warp the representation over larger scale areas. To compensate for the limitation of accumulated error, there are mapping techniques where semi-continuous localization is used. Leonard and Durrant-Whyte [5] and Feng Lu and Evangelos E. Milios [6] employ such methods. But even with these methods, the uncertainties of a robot can never be eliminated, especially when dealing with rough terrains or unstructured regions.

It is not always beneficial to keep metric relations over large scales (since the relation are usually erroneous). A better alternative might be to provide topo- 
logical or qualitative relations over such extents, while storing metric relations over local areas. Prior work in cognitive science suggests humans use a set of $l o-$ cal reference frames topologically connected to model large scale environment. Yeap [7] shows that a module of the Cognitive Mapping Process can be represented with a Relative Absolute (R-A) model. The model consists of a global representation (referred to as Relative Space Representation, or RSR). The RSR describes a qualitative composition of a sequence of local representations $\{\mathrm{S}(1), \mathrm{S}(2), \ldots\}$ called Absolute Space Representations or ASRs. That is, the global map can be considered as a set of clear and accurate patches of local information linked topologically by fuzzy, semi-unknown areas (Figure 1).

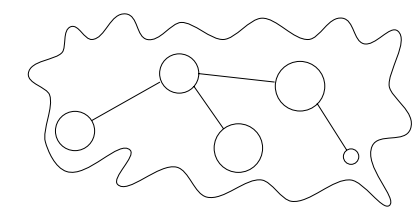

Figure 1: Global map composed of a set of local maps. Circles represent metrically accurate local maps.

Similar to the cognitive approach, there has been work in creating topological-metric maps for mobile robots [1, 8]. Kuipers and Byun [1] develop a mapping and exploration strategy based on both qualitative and quantitative components. Their method considers distinctiveness measures in terms of certain pre-defined sensory criteria.

\section{Islands of Reliability and Topology}

In this work, the environment is represented by a set of accurate local maps. Each local map is built using its own local reference frame and modelling method. In theory, any sensory system and modelling method can be used to create a local map. The local maps are referred to as islands of reliability. When the robot lies within the vicinity of an island, it has the potential to perform local computational tasks involving real world data and stored data with accurate results. The environment is represented by a set of these islands where each island forms an independent computational component of the global map.

The islands of reliability are organized in a topological structure. They form nodes of a topological model of the world, i.e., a graph $[1,9,2]$.

$$
\begin{aligned}
& M=\{V, E\} \\
& V=\left\{v_{0}, v_{1}, \ldots, v_{i}\right\} \\
& v_{i}=\{L, T\} \\
& E=\left\{e_{1}, e_{2}, \ldots, e_{i}\right\} \\
& e_{i}=\left\{v_{i} v_{j}, S, W\right\}
\end{aligned}
$$

$M$ is the global map, composed of a set of nodes $V$ and a set of edges $E$. A node $v_{i}$ corresponds to a local metric map $L$ and the modelling type $T$ used to build the local map. An edge $e_{i}$ corresponds to a set of instructions $S$ describing the node connection, and a weight $W$.

By using separate local reference frames, we avoid the need to perform large-scale error integration. Metric data is gathered only at local areas. At each of these areas, data is mapped using a separate reference frame, forming independent local maps (islands of reliability). The global map consists of a set of these local maps distributed about the environment. The distribution depends on where relevant data exists. That is, where computational tasks involving real world data and a priori data need to be performed. Links between adjacent nodes are provided to describe the connectivity. These links may disconnect metric relations between the nodes. Nevertheless, it is not necessary to keep these relations since over large scales they are erroneous due to accumulated error.

\section{Task Based Distinctiveness Measure}

The topological-metric map, in the form of equation 1 , decomposes the environment into qualitative and quantitative components. Without knowledge of what goal the map is intended for, a configuration for this model cannot be determined. Maps must be tagged with a task. Once the task is defined, the task itself can be decomposed into qualitative and quantitative components. The quantitative components (components requiring computations involving a priori metric data and real world data) and qualitative components (high level instructions not requiring a priori metric data of current environment features) define the configuration of the map. Areas relevant to the computational components are metrically mapped while the qualitative components define their relation.

The topological-metric model described is a general one and can be used for many different mapping goals. So long as the map is tagged with a task, we can decompose the environment at areas computationally relevant to the task and at uninteresting areas. Where 
those areas lie can be determined by a distinctiveness measure:

Definition: The distinctiveness measure $R$ for $a$ computational task $T$ at an area $A$ is measure of how well the features at area $A$ allow the accomplishment of the task $T$.

Given a computational task, we can evaluate areas of the environment by examining if the task can be accomplished properly. Areas that show success are labelled with a high distinctiveness measure and areas that fail are labelled with a low distinctiveness measure.

\section{$5 \quad$ Navigation Map}

The first step in constructing the topological-metric model is to determine the constraints for the spatial distribution of the islands of reliability. This is done in relevance to the computational task they facilitate. In this case, the map we intend to build is a navigation map. In order to properly and safely navigate we should have the ability to localize. This fundamental computational task defines the islands of reliability as localization based maps. Hence, islands are placed at areas where the robot can localize.

It is possible to form the islands of reliability using different sensory systems and modelling methods. Furthermore, there exists many different localization techniques, each with their own strengths and weaknesses. Therefore, the formulation for the distinctiveness measure $R$ may depend on the actual localization scheme and the island models. However, general guidelines can be provided in order to evaluate the environment and select distinctive regions. The general form of the distinctiveness measure $R$ for localization can be described as the following:

$$
R \propto \frac{f(I, \Delta I)\left(1+\sum_{j} \lambda_{j} Q_{j}\right)}{\left(1+\sum_{j} \lambda_{j}\right)}
$$

where $I$ represents the strength of the response of a sensing technique and $\Delta I$ represents the amount of spatial change of that response (which may be expressed as spatial constraint). $f()$ is a function monotonically proportional to to $I$ and $\Delta I . Q_{j}$ is a quality measure specific to the properties of the localization technique and $\lambda_{j}$ is a corresponding weight. That is, to successfully perform localization, there must be sufficient reliable information $I$ subject to spatial variation $\Delta I$ along all degrees of freedom. The addition of ad-hoc quality measures $Q_{j}$, specific to the technique, can improve region selection. However, most of the emphasis is on searching for areas with rich information subject to spatial change (areas providing enough information and low structural ambiguity). A good choice for $f()$ is one of the form $I * \Delta I$, taken along the degree of freedom with the minimum value. Hence, $R$ is large if both $I$ and $\Delta I$ are large in all degrees of freedom.

To complete the model, the topological connectivity of the islands must be defined. The edges form an important component, providing instruction to follow between adjacent nodes. The types of instructions required to accomplish our task are navigation ones. Hence, the edges must include descriptions that allow reliable navigation between connecting nodes, without use of an a-priori metric map. In this regard, the edge of a source node to a target node entail a set of control strategies that navigate the robot from a given position in the source frame to an estimated position in the target frame [1]. The robot can then localize in the target frame using the corresponding computational map dedicated for such a task.

Assuming consistency of error for an exploring robot, we can define the node placement criteria as follows:

$$
\begin{aligned}
& \epsilon>E_{\text {min }} \\
& \max \left(\frac{R_{i}}{T_{i}}\right)>\frac{E_{\min }}{\epsilon}
\end{aligned}
$$

where $\epsilon$ is the estimated accumulated robot error, $E_{m i n}$ is a predefined error bound, $R_{i}$ is the distinctiveness measure for model type $i$ and $T_{i}$ is the acceptable measure threshold for model type $i$. That is, when the exploring robot infers that it is lost (when $\epsilon>E_{\text {min }}$ ) the robot attempts to build an island of reliability using the available models $i$. If the best distinctiveness measure $R_{i}$ normalized by the corresponding acceptance threshold $T_{i}$ is large enough, the island is inserted to the map. Otherwise, the robot continues exploring, searching for a good area. Note that the more lost the robot is the more sensitive it is to accept an island.

\section{Local Map Perception}

This section deals with the localization and modelling technique used to build the islands. The method used is sonar based and is described in the papers by G. Dudek and P. MacKenzie [3] and [10].

In order to perform localization, a model is constructed of how sensory data varies as a function of the robots position. The model is built by fitting line primitives to sensory data. The line fitting method is done in several steps. First, a spatial clustering algo- 
rithm is employed to determine groups of neighbouring points that correspond to a potential line segment. Then, by using a line fitting procedure, a fitted line segment is used to model each cluster. This results is a line segment model as the environment representation.

The pose estimation problem is formulated as an optimization problem in terms of the extent map explains observed measurements. It consists of translating over a correction vector to minimize the difference between new sensory data and existing line segment; the correction vector is calculated as a weighted sum of the error vectors between new sensory data and existing wall segments.

\section{Local Map Distinctiveness Measure Criteria}

A good distinctiveness measure for the sonar based technique is one that rates highly areas well constrained by near line segments of significant length. Furthermore, it is desirable that the line model shows similar orthogonal constraints for both D.O.F., allowing equal localization confidence and keeping the error bound round. In the extreme case, parallel lines would result in ambiguities along one dimensions and will not provide enough information to adapt the full potential of the localization method. For the line model method, the distinctiveness measure $R$ at a point $p=(x, y)$ over a square area $(2 \epsilon)^{2}$ can be calculated as:

$$
R(p, \epsilon)=N(p, \epsilon) \int_{y-\epsilon}^{y+\epsilon} \int_{x-\epsilon}^{x+\epsilon} \frac{F(p)(1+Q(p))}{2} \delta x \delta y
$$

where,

$$
\begin{aligned}
& F(p)=\operatorname{Min}\left[C_{\perp}(p), C_{\|}(p)\right] \\
& N(p, \epsilon)=\frac{1}{\int_{y-\epsilon}^{y+\epsilon} \int_{x-\epsilon}^{x+\epsilon} \delta x \delta y}
\end{aligned}
$$

$C_{\perp}$ and $C_{\|}$represent $f()$ in equation 2 along two orthogonal D.O.F. $Q$ is a quality measure constraining the localization confidence to remain circular. It is based on the ratio of $C_{\perp}$ and $C_{\|}$, rating the equality of localization confidence about both dimensions. We integrate over a rectangular area defined by $\epsilon$ and normalize.

To describe $C_{\|}$and $C_{\perp}$ we must first derive $I$ and $\Delta I$ in terms of the line model. It is easy to see that the amount of reliable information $I$ is proportional to the amount of visible lines and their distance to the robot. Distant lines provide less reliable information than near ones. Furthermore, a line segment provides strong constraining information $\Delta I$ only along the normal. That is, an orthogonal position change with respect to the line guarantees a sensory measurement change. We define $\Delta I$ to be the orthogonal component of a sensed line segment.

For each line segment, we integrate the strength $I$ and the orthogonal constraint $\Delta I$ to determine the Vector Influence along the normal to the line. We compute the Vector Influence for each visible line segment (in the form of $I * \Delta I$ ) by:

$$
\mathbf{V}_{\mathbf{i}}(\mathbf{p})=\hat{N}_{i} \int_{\Theta} W\left(\mathbf{p p}_{\mathbf{i}}\right) *\left(\hat{N}_{i} \bullet \mathbf{p} \hat{\mathbf{p}}_{\mathbf{i}}\right) \delta \Theta
$$

$V i$ is the orthogonal vector influence for line segment $i$ seen by point $p$ and $\hat{N}_{i}$ is the unit normal of line segment $i$. $\Theta$ sweeps the visible viewing directions from point $p$ to the line segment. Only angles within a reflectance threshold are taken to account in order to simulate specular reflection of real range signals. $p_{i}$ is the intersection point of line segment $i$ and a line emitted from point $p$ along the viewing direction $\Theta$. The constraining relation for $\mathbf{V}_{\mathbf{i}}$ is in essence a projection of the vectors formed from point $p$ to line segment points onto the normal of the line segment. $W(.$.$) expresses the reduced probability of observing$ an object as a function of distance.

$$
W(\mathbf{v})=e^{-k\|\mathbf{v}\|}
$$

$k$ is the decay constant that is determined by the range of sensor confidence.

Once the vector influence is computed for all visible line segments, we choose a reference vector and determine the total number of components parallel and perpendicular to it. These components determine the magnitude of constraint along two orthogonal directions and are calculated as:

$$
\begin{aligned}
& C_{\|}(p)=\sum_{i \text { lines }}\left|\hat{V}_{\text {ref }}(p) \bullet \mathbf{V}_{\mathbf{i}}(\mathbf{p})\right| \\
& C_{\perp}(p)=\sum_{i \text { lines }}\left\|\hat{V}_{\text {ref }}(p) \times \mathbf{V}_{\mathbf{i}}(\mathbf{p})\right\|
\end{aligned}
$$

A good choice for the reference vector is that of largest magnitude vector, since it determines the dominating D.O.F. constraint.

To distinguish good valued from bad ones, we remap them using a sigmoid filter:

$$
C=\frac{C^{m}}{c^{m}+C^{m}}
$$

The cutoff threshold $c$ and the decay rate $m$ can be found empirically. 
We compute a quality measure $Q$ as:

$$
Q(p)= \begin{cases}\frac{C_{\perp}(p)}{C_{\|}(p)} & \text { if } C_{\|}>C_{\perp} \\ \frac{C_{\|}(p)}{C_{\perp}(p)} & \text { otherwise }\end{cases}
$$

$Q$ ranges form 0 to 1 where 1 represents equal orthonormal constraints and 0 represents that only one D.O.F. is constraint.

\section{Results and Discussion}

In this section, we proceed in evaluating the distinctiveness measure by comparing localization error and distinctiveness measure. A good distinctiveness measure should show large values at areas with low error and low values at high error locations. We then construct the topological-metric model using the mapping criteria.

\subsection{Sonar System Measure Evaluation}

A line segment model was manually constructed providing the simulated environment shown in figure $3(\mathrm{~b})$. The distinctiveness measure for the model is plotted in figure 3(a). These results were obtained with the neighbouring area $\epsilon$ set to zero (such that measures consist of only a single point rather that an accumulation of a neighbourhood). It can be seen in the figures that long hallways and distant areas show low measures while regions with good orthogonal constraints (such as intersections and bounding areas) show high measures.

Figure $3(\mathrm{c})$ is a plot of the localization confidence. This plot was generated using a robot controller/simulator. At each position, simulated sonar data was collected, thereafter employing a position offset by a random $\delta$ ranging $10-15 \mathrm{~cm}$. The localization technique was then used to output the position estimation. The error was the difference between the initial position to the estimated one. Confidence is simply $c$-error where $c$ is some constant.

We can seen how the confidence plot is consistent with the distinctiveness measure. Low confidence valleys match the low measure valleys, where there are not enough constraints. Figure 2 shows the accumulated residual plot between the distinctiveness measure and localization confidence. There are not many data points with residual greater than 0.4 (about $25 \%$ of the data).

One significant difference between the plots is that the localization confidence exhibits sharp drops while

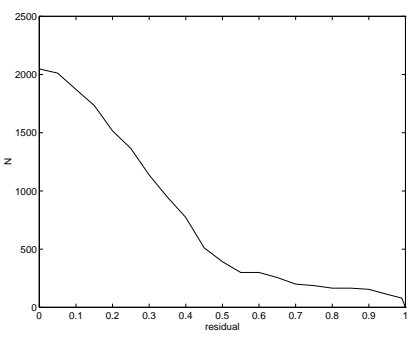

Figure 2: Residual plot for figure 3(a) and 3(c), there are $\mathrm{N}$ data points with differences greater than residual.

the distinctiveness measure undergoes smooth decays. This is due to the fact that the localization technique is only accurate within a region of convergence. Once the robot moves beyond that region the solution becomes completely incorrect; there is a narrow midground. The analytic distinctiveness measure, on the other hand, is a smooth continuous function. Hence, the variational inconsistencies between the plots are consistent with the theory.

Keeping this in mind, a mapping threshold can be determined by the intersection point of the distinctiveness measure and the confidence cutoff region. Areas where the measure is less than the threshold are not reliable and should not be mapped but areas displaying larger measures are good candidates. Furthermore, the filter parameters can be determined more accurately by forming an optimization criterion minimizing the residual plot. Searching the parameter space to minimize the difference between distinctiveness measure and localization confidence can improve results.

\subsection{Mapping with Line Segment Model}

The goal of this experiment was to explore an environment and follow the mapping criterion (equation 3) to build a topological-metric representation as in equation 1. A large scale simulated environment was built using the robot controller/simulator. A simulated robot explored the environment using a pre-defined set of control strategies extracted from a simple hall following procedure [11]. (Although, more complex and autonomous exploration strategies may be used.)

The robot is begins at a starting node and follows the control strategies. Once the robot infers it is lost, it attempts to to build a line segment model (a node). The node building routine is a simple one that collects data at four corners within the visibility range. (More advanced node building routines can easily be 


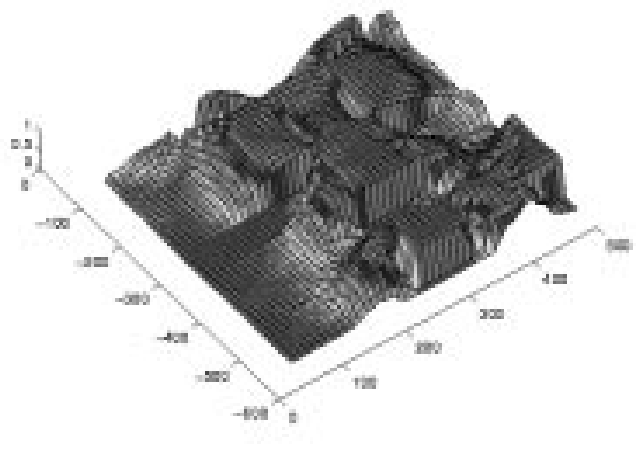

(a)

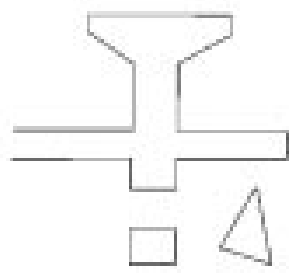

(b)

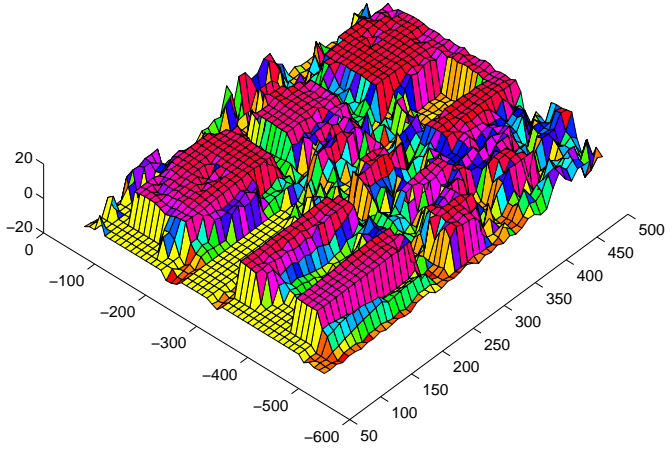

(c)

Figure 3: Sonar based distinctiveness measure 3(a) and localization confidence 3(c) for a simulated environment 3 (b) with hallways, intersections and bounding regions.

incorporated to the structure.) While collecting data, the robot intermittently calculates the distinctiveness measure to check the region. If the region is acceptable, the new node is added to the map, with a link to the previous node and the control strategies that were followed. Figure 4(a) shows the simulated environment and the exploration path and figure $4(\mathrm{~b})$ shows the topological-metric map. It can be seen that the robot built nodes at well constrained areas. Areas such as hallways were not included in the map since they lack in information along one degree of freedom. The topological-metric model (figure 4(b)) shows the line segment models within the nodes. Each node represents a separate local map with its own co-ordinate system, no global reference is used. The spatial arrangement of nodes in the figure are only set for clarity. The links between nodes in the figure describe the topology and include a compilation of the control strategies used in the exploration step.

Each control strategy is initiated at coordinates $(x, y, \Theta)=(0,0,0)$ in the corresponding reference frame. Therefore, to navigate from node to node, the robot must first localize at $(0,0,0)$ then follow the control instructions. The control strategies used form a simple approach for traversing the environment. Although they are not purely qualitative controls, they do form instructions that navigate the robot from one node to the next without the need of a priori data gathered at the links. Furthermore, they can be replaced by purely qualitative high level controls strategies such as follow wall to next node (or such as those listed in [1]).

The experiment was also executed in a real environment. Figurer 5(a) shows a real environment and 5(b) shows the topological-metric representation of that environment. Table 1 contains the links of the topological map. The robot was set to extract the first 3 nodes of the hallways near our laboratory.

\begin{tabular}{|r|r|}
\hline Link & Control Strategies \\
\hline 0 & Go 125, Rot 270, Go 652 \\
1 & Rot 270, Go 28, Rot 90, Go 832 \\
\hline
\end{tabular}

Table 1: Control strategies for inter-node navigation

\section{Conclusion}

This paper describes an important step in the creation of large scale maps that combine both metric and topological knowledge. Specifically, we describe how the locations of individual localization regions or islands of reliability can be selected using a distinctiveness measure. The distinctiveness measure showed consistency with localization confidence, making it a good measure of environment quality for localization. We then examine the mapping criterion using a simulated and a real environment to form the topologicalmetric map. The paper was put in context to high level mapping goals that requires the framework of 


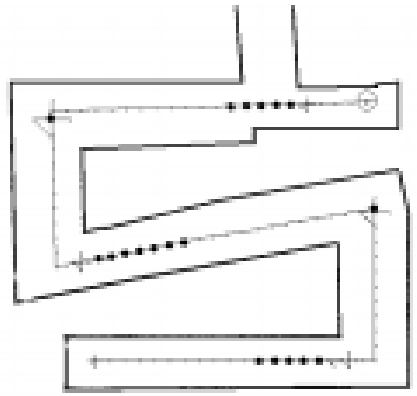

(a)

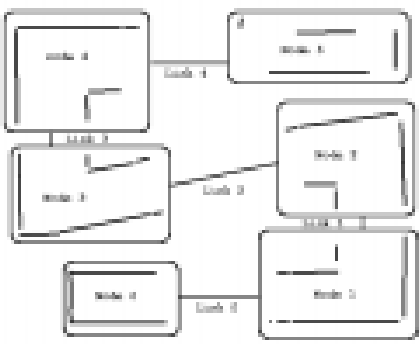

(b)

Figure 4: Simulated map and the exploration path 4(a) and the topological-metric map 4(b). Large dots in the exploration path show where the robot attempted to place an island but immediately failed. The full star-like paths show where the robot had successfully completed to build a island. The partial star-like paths show an attempt that partially built a node but was dismissed due to later discovery of low distinctiveness measure.

environment evaluation.

\section{References}

[1] B. Kuipers and Y-T. Byun, "A robot exploration and mapping strategy based on a semantic hierarchy of spatial representations", Robotics and Autonomous Systems, vol. 8, pp. 47-63, 1991.

[2] Gregory Dudek, Michael Jenkin, Evangelos Milios, and David Wilkes, "A taxonomy for multi-agent robotics", Autonomous Robots, vol. 3, pp. 375-397, 1996.

[3] Paul MacKenzie and Gregory Dudek, "Precise positioning using model-based maps", in IEEE International Conference on Robotics and Automation, San Diego, California, May 1994, pp. 1615-1621.

[4] G. Dudek and C. Zhang, "Vision based robot localization without explicit object models", in IEEE Conference on Robotics and Automation, Minneapolis, MN, May 1996, pp. $76-82$.

[5] John J. Leonard and Hugh F. Durrant-Whyte, "Mobile robot localization by tracking geometric beacons", IEEE Transactions on Robotics and Automation, vol. 7, no. 3, pp. 376-382, June 1991.

[6] F. Lu and E. Milios, "Optimal global pose estimation for consistent sensor data registration", in IEEE International Conference on Robotics and Automation, 1995, pp. 93-100.

[7] W. Yeap, "Towards a computational theory of cognitive maps", Artificial Intelligence, vol. 32, pp. 297-360, 1988.

[8] S. Thrun, "Learning metric-topological maps for indoor mobile robot navigatio n", in AI Journal 99(1), 1998, pp. 21-71.

[9] Gregory Dudek and John K. Tsotsos, "Shape representation and recognition from curvature", in Proceedings of the 1991 Conference on Computer Vision and Pattern Recognition, Maui, Hawaii, June 1991, pp. 35-41, IEEE.
[10] Paul MacKenzie, "Mobile robot localization using modelbased maps", Master's thesis, Dept. of Electrical Engineering, Montréal, Québec, 1994.

[11] Philippe Ciaravola, "Maze exploration", Tech. Rep., Centre for Intelligent Machines, McGill University, 1996. 


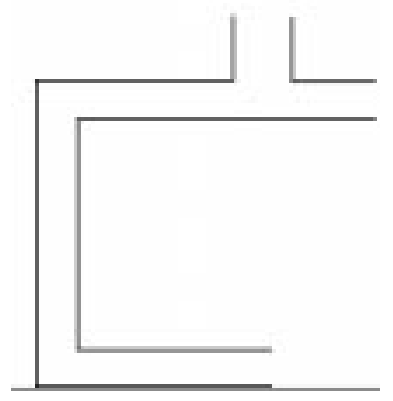

(a)

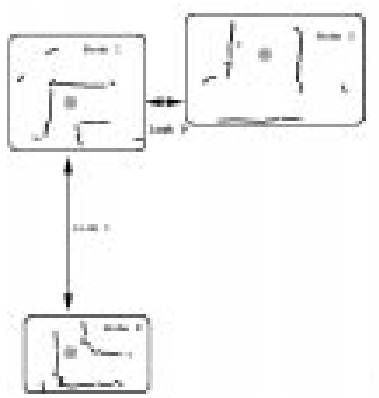

(b)

Figure 5: A map of a real environment 5(a) and the topological-metric map 5(b). The dots in the topological map show the origin of the local frame. 